\title{
Erratum to: The Influence of Stabilized Deconjugated Ursodeoxycholic Acid on Polymer-Hydrogel System of Transplantable NIT-I Cells
}

\author{
Armin Mooranian ' • Rebecca Negrulj' • Hani Al-Salami '
}

Published online: 24 February 2016

(C) Springer Science+Business Media New York 2016

\section{Erratum to: Pharm Res \\ DOI 10.1007/sI 1095-016-1863-y}

In the article entitled, "The Influence of Stabilized Deconjugated Ursodeoxycholic Acid on Polymer-Hydrogel System of Transplantable NIT-1 Cells," appearing in Pharm. Res. (2016), the following syntactic corrections should be made to the Introduction. The changes do not affect the results or discussion of the article.

In the second paragraph of this section, the original document states:

.... One of the most commonly used polymer in cell microencapsulation is sodium alginate $(S A)$ due to its good biocompatibility and biodegradability characteristics (I3). However, encapsulating cells using only SA for cell transplantation produces microcapsules with many limitations. The limitations of the microcapsules are mainly poor mechanical strength, weak membrane, and uneven membrane porosity and density, which result in membrane deformation, cell leakage and sudden rupture of microcapsules (I4). In order to overcome the limitations of SA-microcapsules, additional excipients have been incorporated into the microencapsulating formulation. Various excipients have been shown to enhance the physical structure and mechanical strength of the microcapsules, however, cell viability remains low, specially in the long-term (I5)...

The online version of the original article can be found at $h \mathrm{ttp}: / / \mathrm{dx}$.doi.org/I0. |007/s | |095-0 | 6-1863-y.

Hani Al-Salami

hani.al-salami@curtin.edu.au

Biotechnology and Drug Development Research Laboratory, School of Pharmacy, Curtin Health Innovation Research Institute, Curtin University, Perth, WA, Australia
The revised version should read:

... Sodium alginate $(S A)$ is widely used for cell encapsulation. This is due to its good compatibility properties (13). However, by itself, SA does not produce the best transplantable microcapsules due to limitations, including weak structure and thin membrane with increased porosity, which may result in cell leakage and sudden breakage of the microcapsule (14). The limitations may be overcome by adding new excipients that can provide mechanical support; however, cell viability seems to remain low, especially in long-term transplantation ( 15$) \ldots$

In the fourth paragraph of this section, the original document states:

...Ideally, an excipient is required that has been shown to support microcapsules' physical structure and mechanical strength and also support cell viability, functionality and metabolism as well as possess antiinflammatory effects. An example of such an excipient is the tertiary bile acid, ursodeoxycholic acid (UDCA). In a recent study in our laboratory, UDCA incorporation into SA microcapsules has shown promise in supporting $\beta$-cell growth (17)...

The revised version should read:

...A good excipient will be one which has shown to support cell survival and mass, and also enhance the microcapsules' topographic properties. Ideally, the excipient will exert biological and anti-inflammatory effects to support further cell mass. Ursodeoxycholic acid (UDCA) is a bile acid that we have shown to be able to exert some beneficial effects on microencapsulated $\beta$-cells that are of significant importance $(17) \ldots$ 